\title{
Irregularities of ytterbium under high pressure
}

\author{
Y. C. Zhao, ${ }^{*}$ F. Porsch, and W. B. Holzapfel \\ Fachbereich Physik, Universität-GH-Paderborn, D33095 Paderborn, Germany
}

(Received 30 August 1993)

\begin{abstract}
Phase transitions of the rare-earth metal ytterbium were studied under pressure up to $70 \mathrm{GPa}$ at room temperature with an energy-dispersive $\mathrm{x}$-ray-diffraction technique using synchrotron radiation. A new phase transition $\mathrm{hcp} \rightarrow \mathrm{fcc}(\mathrm{II})$ was observed at $57 \mathrm{GPa}$. The phase sequence with pressure up to $70 \mathrm{GPa}$ is $\mathrm{fcc}(\mathrm{I}) \rightarrow \mathrm{bcc} \rightarrow \mathrm{hcp} \rightarrow \mathrm{fcc}(\mathrm{II})$. The hcp and fcc(II) phases show some similarity in their compression behavior to the lower pressure phases of praseodymium which is considered as first evidence for a strong hybridization between $f$ electrons and conduction electrons.
\end{abstract}

Ytterbium $(\mathrm{Yb})$ is a member of the rare-earth metals or lanthanides (lanthanum though lutetium). However, it behaves irregular in comparison with other "regular" (trivalent) members of the lanthanide series due to its special electronic configuration. In solid state at ambient conditions, $\mathrm{Yb}$ is considered as divalent with an electronic configuration close to $4 f^{14}(5 d 6 s)^{2}$, whereas the other trivalent lanthanides are characterized by $4 f^{n}(5 d 6 s)^{3}$ with $n$ going from 0 for lanthanum to 14 for lutetium. The special electronic configuration of $\mathrm{Yb}$ results first of all in a larger atomic volume at ambient condition and, furthermore, in a special sequence of phase transitions at ambient pressures and elevated temperatures as well as under pressure. In previous high-pressure studies ${ }^{1-3}$ up to $39 \mathrm{GPa}$ at ambient temperature, only the structural sequence $\mathrm{fcc} \rightarrow \mathrm{bcc} \rightarrow \mathrm{hcp}$ was observed in contrast to $\mathrm{hcp} \rightarrow \mathrm{Sm}$-type $\rightarrow d \mathrm{hcp} \rightarrow \mathrm{fcc} \rightarrow$ distorted-fcc for the regular (trivalent) lanthanides. ${ }^{4,5}$

Theoretical considerations ${ }^{6}$ as well as $\mathrm{x}$-ray absorption near-edge structure (XANES) experiments ${ }^{7}$ gave very strong indications for a change in the number of electrons per atom in the conduction band of $\mathrm{Yb}$ from almost 2 to almost 3 in the pressure range from 0 to $30 \mathrm{GPa}$, and theorists expected, therefore, 8,9 that $\mathrm{Yb}$ under strong compression should ultimately follow also the structural sequence of the regular lanthanides. In this sense, the hcp phase could be considered as the first phase of the common structural sequence for all the regular trivalent lanthanides. Studies of the pressure-volume dependence by shock wave experiments ${ }^{10,11}$ resulted in an equation of state (EOS) for ambient temperature which was compatible with regular trivalent behavior above roughly 30 $\mathrm{GPa}$; however, structural information could not be derived from these studies. The present study, therefore, gives the first information on the structural behavior in the higher-pressure range from 40 to $70 \mathrm{GPa}$ at room temperature.

The present experiments were carried out with energy dispersive x-ray diffraction (EDXRD) using synchrotron radiation at HASYLAB, DESY (Hamburg). The details of this experimental station have been described previously. ${ }^{12}$ High pressure was generated by a beveled diamond anvil cell ${ }^{13}$ (DAC) using an inconel gasket with a $100 \mu \mathrm{m}$ hole. The collimator in front of the DAC was set to 60 $\mu \mathrm{m}$. Silicone oil was used as pressure transmitting medi- um which prevents also oxidation of the sample during sample loading. Fine ruby powder was loaded in the DAC as pressure sensor and the nonlinear rubyluminescence scale ${ }^{14}$ was adopted for the pressure determination. The $\mathrm{Yb}$ sample material was provided by $\mathrm{K}$. A. Gschneider, Jr., with chemical analysis giving the major impurity contents in atomic ppm as follows: 30 for iron, 30 for all other lanthanides, 304 for oxygen, and 1384 for hydrogen.

Figure 1 shows typical EDXRD pattern of $\mathrm{Yb}$ under pressure, where the lattice spacings $d$ are related to the measured energies $E$ by $E d=66.2528 \mathrm{keV} \AA$. The peak labeled $G$ is from the gasket. The peak labeled $X$ appears in all spectra with nearly constant relative intensity, and shifts with pressure less than other diffraction lines. Hence the peak $X$ does not belong to pure Yb metal. The

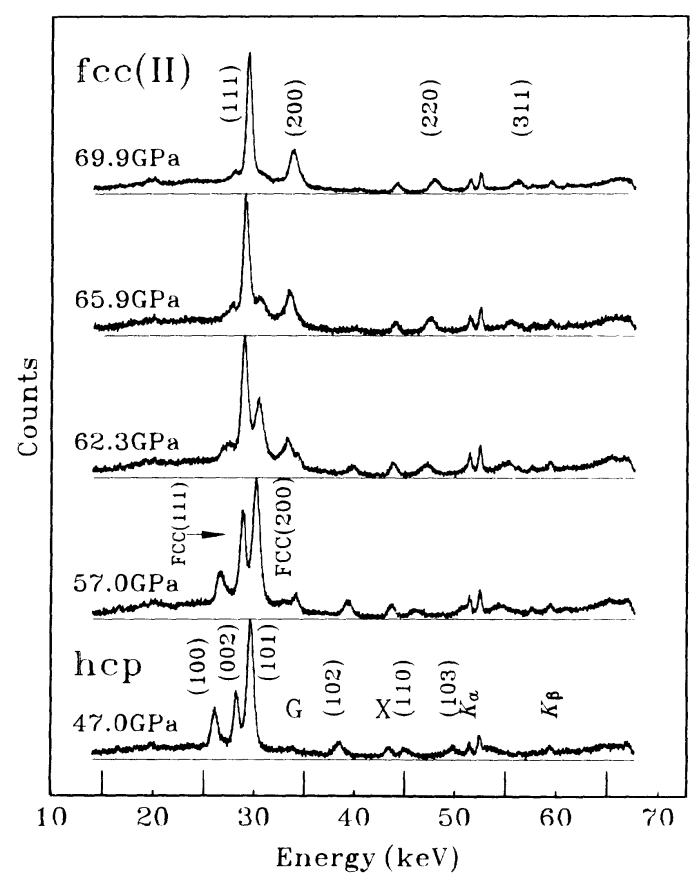

FIG. 1. Energy dispersive $\mathrm{x}$-ray-diffraction spectra of $\mathrm{Yb}$ showing the evolution of the diffraction patterns for the $\mathrm{hcp} \rightarrow \mathrm{fcc}(\mathrm{II})$ transition under isothermal compression at room temperature. 
lattice spacing of this peak varies from $0.1587 \mathrm{~nm}$ at 14.5 GPa to $0.1501 \mathrm{~nm}$ at $69.9 \mathrm{GPa}$ and thus can neither be attributed to any diffraction line of $\mathrm{YbO}$ according to the volume-pressure relationship for the only presently known phase ${ }^{15}$ and hence remains unexplained. All structural changes below $39 \mathrm{GPa}$ noticed in the present study are in agreement with the previous studies. ${ }^{1-3}$ At higher pressures, however, a new phase transition $\mathrm{hcp} \rightarrow \mathrm{fcc}$ starts at about $57 \mathrm{GPa}$ where the relative volume $V / V_{0}=0.40, a=0.287 \mathrm{~nm}$ and $c=0.459 \mathrm{~nm}$ for the hcp phase, and $a=0.403 \mathrm{~nm}$ for the fcc phase, when the (111) and (200) lines of the new fcc phase appear. The difference of the atomic volume between these two phases is smaller than the experimental uncertainty $(<1 \%)$. With increasing pressure, the intensities for fcc lines (111), (200), (220), and (311) increase and the hcp lines disappear. In addition, the relative intensities of corresponding diffraction lines substantiate within the typical experimental limits this assignment of fcc structure. In order to distinguish between the two fcc phases below 4 $\mathrm{GPa}$ and above $57 \mathrm{GPa}$, the higher-pressure fcc phase is labeled fcc(II), while the low-pressure phase is labeled $\mathrm{fcc}(\mathrm{I})$. The reverse transition $\mathrm{fcc}(\mathrm{II}) \rightarrow$ hcp for decreasing pressure starts around $45 \mathrm{GPa}$, where $V / V_{0}=0.41$, $a=0.410 \mathrm{~nm}$ for the fcc(II) phase, and almost finishes around $37 \mathrm{GPa}$. Thus, the present best estimate for the equilibrium transition pressure is 53(10) $\mathrm{GPa}$ and the atomic volume is $0.017(1) \mathrm{nm}^{3}$.

Figure 2 shows the variation of atomic volume with pressure for $\mathrm{Yb}$. Some previous EOS data for $\mathrm{Yb}$ from static measurements ${ }^{3}$ are also included in this figure to provide sufficient data for a smoothed representation of the EOS curve especially for the close-packed highpressure phases hcp and fcc(II) together. Since theoretical consideration and experimental XANES studies on the electronic configuration of $\mathrm{Yb}$ under pressure gave strong evidence for the promotion of almost one complete $f$ electron per atom to the conduction band in the pressure range from 0 to $30 \mathrm{GPa}$, it is useful to compare the present EOS data for the region above $30 \mathrm{GPa}$ with the $P-V$ variation for hypothetical "regular" trivalent $\mathrm{Yb}$ as it is given by the average of the EOS curves for Tm

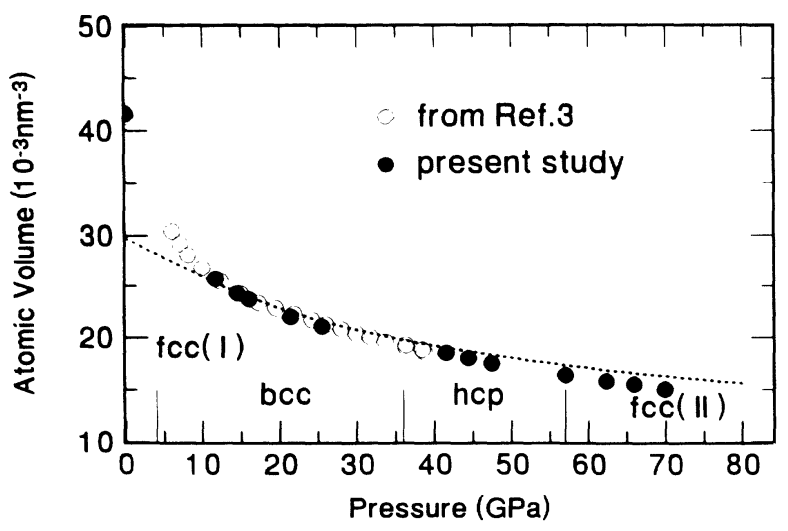

FIG. 2. Experimental isothermal pressure-volume data of Yb. Previous data (Ref. 3) are presented by open circles and the present data by solid circles. The dotted curve is the $P-V$ variation of the hypothetical regular trivalent $\mathrm{Yb}$. and $\mathrm{Lu}$, and represented by the dotted curve in Fig. 2. While the volume for hypothetical regular trivalent $\mathrm{Yb}$ is reached at about $15 \mathrm{GPa}$, further compression results, however, in a stronger volume decrease than expected for regular trivalent $\mathrm{Yb}$ or, in other words, $\mathrm{Yb}$ becomes softer than expected. A similar behavior is observed also for a few other trivalent lanthanides $(\mathrm{Ce}, \mathrm{Pr})$ and actinide elements (Am-Cf) when $f$ electrons start to delocalize or start to contribute to bonding by strong hybridization with the conduction electrons. ${ }^{5}$

If one compares the structural sequence hcp $\rightarrow$ fcc(II) with the well-known sequence hcp $\rightarrow$ Sm-type $\rightarrow$ $\mathrm{dhcp} \rightarrow \mathrm{fcc} \rightarrow$ distorted-fcc for the regular trivalent lanthanides, one can notice also that the intermediate structures Sm-type and $d$ hcp are missed. Hence the phase transition hcp $\rightarrow \mathrm{fcc}$ (II) does not fit to the behavior of the regular lanthanides. In addition, the measured $c / a$ values for the hcp phase in $\mathrm{Yb}$ is 1.60 in contrast to $1.57-1.59$ of hcp phases in the trivalent lanthanides, and thus are closer to the ideal $c / a$ value (1.633) of the hcp lattice than in the regular trivalent lanthanides.

Furthermore, the common sequence of phase transitions in the regular trivalent lanthanides can be characterized by radius ratios ${ }^{5,16} R_{\mathrm{wS}} / R_{5 p}$, where $R_{\mathrm{wS}}$ is the Wigner-Seitz radius and $R_{5 p}$ represents the radius of the $5 p$ electron orbital of trivalent ion of lanthanide given in Ref. 17. At the equilibrium transition pressure for the $\mathrm{hcp} \rightarrow \mathrm{fcc}$ (II) transition in $\mathrm{Yb}$ (at $53 \mathrm{GPa}$ ), $R_{\mathrm{ws}}=0.159$ $\mathrm{nm}$ and thus $R_{\mathrm{wS}} / R_{5 p}=2.48$, whereas the common value of $R_{\mathrm{wS}} / R_{5 p}$ that characterizes the $d$ hcp $\rightarrow$ "fcc" transition of the regular trivalent lanthanides ${ }^{5,16}$ is 2.46(4). Thereby "fcc" stands for either the fcc structure or the distorted-fcc structure which is directly formed from dhcp at room temperature in the case of the heavier lanthanides (Tb-Lu). Thus, a comparison of the $R_{\text {wS }} / R_{5 p}$ value for the hcp $\rightarrow$ fcc(II) transition of $\mathrm{Yb}$ with that for the regular lanthanides at the $d$ hcp $\rightarrow$ "fcc" transition shows that the difference is within the typical scatter of these values for the other (heavier) lanthanides; however, the appearance of the superlattice diffraction lines for this distorted-fcc phase of regular trivalent lanthanides is not observed.

Finally one can evaluate the EOS data for the two close-packed high-pressure phases hcp and fcc(II) together in the scaling scheme extensively used in a recent comparison of EOS data for regular and irregular lanthanides with actinides under pressure. ${ }^{18,19}$ Thereby the pressure $P$ was scaled by the pressure of the corresponding Fermi gas pressure $P_{\mathrm{FG}}=a_{\mathrm{FG}}(Z / V)^{5 / 3}$ where $a_{\mathrm{FG}}=23.37$ $\mathrm{MPa} \mathrm{nm}{ }^{5}, Z$ is the atomic number, and $V$ stands for the atomic volume represented by the volume of the WignerSeitz cell. If one uses in addition the scaled atomic radius $\sigma=[3 Z V /(4 \pi)]^{1 / 3}$ with its value $\sigma_{0}$ at ambient condition, one can represent the EOS data in a scaled form by a plot of the quantity $\eta=\ln \left(P / P_{\mathrm{FG}}\right)-\ln \left(1-\sigma / \sigma_{0}\right)$ versus $\sigma$ as shown in Fig. 3, which allows for direct comparison of EOS data for different elements and especially within the lanthanide and actinide families as discussed in detail previously. ${ }^{18-20}$ More specifically, the EOS data of all the regular trivalent lanthanides are presented in this scaled form within the given experimental error just 
by a common relation, which is represented in Fig. 3 by the slightly curved dashed line and starts at the common value $\sigma_{0}=0.792(3) \mathrm{nm}$ for all the regular trivalent lanthanides. ${ }^{15}$ Besides the irregular lanthanides $(\mathrm{Ce}, \mathrm{Eu}$, and $\mathrm{Yb}$ ) also the elements $\mathrm{La}$ and $\mathrm{Pr}$ show already at rather low pressures $(<20 \mathrm{GPa})$ significant deviations from this common behavior as illustrated by the dashdotted line just for Pr in Fig. 3. The data for the bcc phase of $\mathrm{Yb}$ already discussed in Ref. 18 are represented here again by the open circles.

Since the values of $\eta$ in this representation depend on the value for $\sigma_{0}$ used in the scaling, one must be careful with the interpretation of data for high-pressure phases like the data for $\mathrm{Yb}$ in the hcp and fcc(II) phases represented in Fig. 3 by open diamonds and squares, which correspond to the use of $\sigma_{0}=0.883 \mathrm{~nm}$ for the low-pressure (divalent) fcc(I) phase. For a comparison with the regular trivalent behavior, the use of the common trivalent value for $\sigma_{0}=0.792(3) \mathrm{nm}$ is more appropriate for these high-pressure phases, corresponding to an extrapolation of the EOS for the hypothetical regular trivalent $\mathrm{Yb}$ to ambient condition. Scaled with this $\sigma_{0}$ value, the EOS data of the close-packed high-pressure phases of $\mathrm{Yb}$ (indicated by solid diamonds and squares in Fig. 3) show some similarity to the irregular behavior of Pr before its volume collapse transition. On the basis of the EOS behavior of $\mathrm{Yb}$ one is therefore also led to the conclusion, that $\mathrm{Yb}$ in these close-packed high-pressure phases does not show typical behavior of a simple or regular trivalent lanthanide metal, but is effected like $\mathrm{Pr}$ in its low-pressure phases already by significant contributions of hybridization between the still almost localized $f$ electrons with the conduction electrons, in other words, by weak $f$ electron bonding.

Note added in proof. Our latest measurements on $\mathrm{Yb}$ up to $90 \mathrm{GPa}$ confirm that the peak marked with $X$ results not from the $\mathrm{Yb}$ sample since it does not occur in

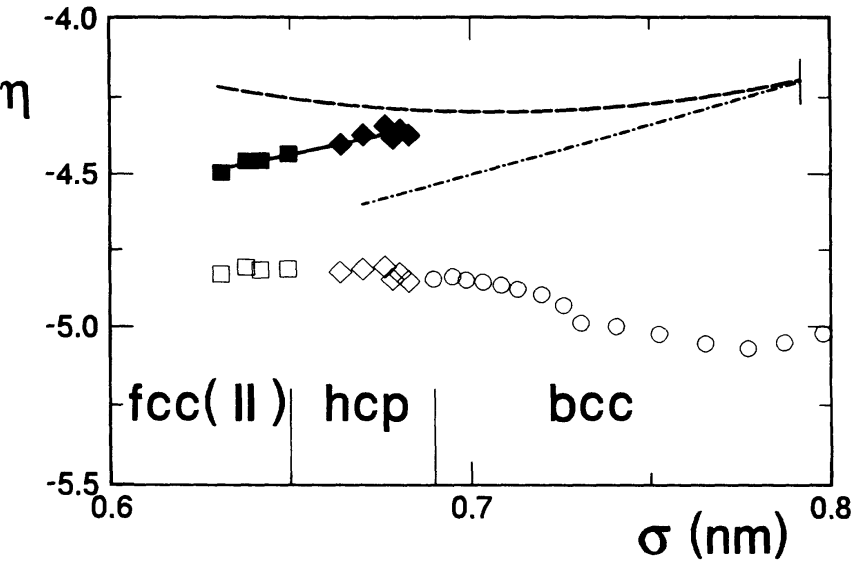

FIG. 3. Compression data of $\mathrm{Yb}$ in $\eta-\sigma$ representation with $\eta=\ln \left(\mathrm{P} / \mathrm{P}_{\mathrm{FG}}\right)-\ln \left(1-\sigma / \sigma_{0}\right)$. The experimental compression data for hcp and fcc(II) scaled with the value of $\sigma_{0}=0.792(3)$ $\mathrm{nm}$ for the regular trivalent lanthanides are indicated by solid diamonds and squares, respectively. The data for hcp and fcc(II) scaled with the divalent value of $\sigma_{0}=0.883 \mathrm{~nm}$ are indicated by the open diamonds and squares. The dashed curve indicates the EOS for the hypothetical regular trivalent $\mathrm{Yb}$ and the dash-dotted line the compression behavior of Pr before its volume-collapse transition. The data for the bcc phase taken from literature ${ }^{3,18}$ are shown by open circles.

these new measurements, and the stability of the phase fcc(II) extends up to $90 \mathrm{GPa}$.

This work is supported in parts by the Deutsche Forschungsgemeinschaft, the Bundesministerium für Forschung und Technologie and HASYLAB, DESY. The authors would like to thank Professor K. A. Gschneider, Jr. for providing the sample material and W. Sievers, O. Schulte, M. Winzenick, and J. Otto for assistance in the experiment. One of the authors (Y.C.Z.) is indebted to Alexander von Humboldt-Stiftung for financial support.
*Permanent address: Beijing Synchrotron Radiation Facility, P.O. Box 918, 2-7, Beijing 10039, People's Republic of China.

${ }^{1}$ W. B. Holzapfel, T. G. Ramesh, and K. Syassen, J. Phys. (Paris) Colloq. 40, C5-390 (1979).

${ }^{2}$ K. Takemura and K. Syassen, J. Phys. F 15, 543 (1985).

${ }^{3}$ W. A. Grosshans, Ph.D. thesis, University of Paderborn, 1987.

${ }^{4}$ A. Jayaraman, Phys. Rev. A 135, 1056 (1964), D. B. McWhan, Science 176, 751 (1972); K. A. Gschneider, Jr. and F. W. Calderwood, in Handbook on the Physics and Chemistry of Rare Earths, edited by K. A. Gschneider, Jr. and L. Eyring (Elsevier, New York, 1986), Vol. 8, p. 1.

${ }^{5}$ U. Benedict, W. A. Grosshans, and W. B. Holzapfel, Physica 144B, 14 (1986) and references therein.

${ }^{6} \mathrm{~J}$. F. Herbst and J. W. Wilkins, in Handbook on the Physics and Chemistry of Rare Earths, edited by K. A. Gschneider, Jr., L. Eyring and S. Hüfner (Elsevier, New York, 1987), Vol. 10, p. 321.

${ }^{7}$ K. Syassen, G. Wortmann, J. Feldhaus, K. H. Frank, and G. Kaindl, Phys. Rev. B 26, 4745 (1982).

${ }^{8}$ B. Johansson and A. Rosengren, Phys. Rev. B 11, 2836 (1975).

${ }^{9}$ H. L. Skriver, Phys. Rev. Lett. 49, 1768 (1982); Phys. Rev. B 31, 1909 (1985).

${ }^{10}$ W. H. Gust and E. B. Royce, Phys. Rev. B 8, 3595 (1973).
${ }^{11}$ W. J. Carter, J. N. Fritz, S. P. Marsh, and R. G. McQueen, J. Phys. Chem. Solids 36, 741 (1975).

${ }^{12}$ For example, W. A. Grosshans, E.-F Düsing, and W. B. Holzapfel, High Temp. High Pressure 16, 539 (1984).

${ }^{13}$ K. Syassen and W. B. Holzapfel, Europhys. Conf. Abstr. 1A 75 (1975); W. B. Holzapfel, in High Pressure Chemistry, edited by H. Kelm (Reidel, Boston, 1978), p. 177.

${ }^{14}$ H. K. Mao, P. M. Bell, J. W. Shaner, and D. J. Steinberg, J. Appl. Phys. 49, 3276 (1978).

${ }^{15}$ A. Werner, H. D. Hochheimer, A. Jayaraman, and J. M. Leger, Solid State Commun. 38, 325 (1981).

${ }^{16}$ T. Krüger, B. Merkau, W. A. Grosshans, and W. B. Holzapfel, High Pressure Res. 2, 193 (1990).

${ }^{17}$ J. T. Waber and D. T. Crommer, J. Chem. Phys. 42, 4116 (1965).

${ }^{18}$ W. A. Grosshans and W. B. Holzapfel, Phys. Rev. B 45, 5171 (1992).

${ }^{19}$ W. B. Holzapfel, Physica B190, 21 (1993), and references therein.

${ }^{20}$ W. B. Holzapfel, Europhys. Lett. 16, 67 (1991); in Molecular Solids Under Pressure, edited by R. Pucci and G. Piccitto (North-Holland, Amsterdam, 1991), p. 61. 